\title{
Prandtl's universal velocity distribution law in streams: a consequence of brownian nature of turbulence at dynamic equilibrium
}

\begin{abstract}
The great advances in mathematics, physics and chemistry that happened from the second half of the twentieth century have radically changed the views on many of the fundamental questions of Science and its application to the demands of society. One of them is turbulence in fluids, the great challenge of all times. Many of its aspects remaining in the mystery, but its essence have been captured in general, especially by the theory of chaos and "strange attractors." However, the role of thermodynamics is less known, especially the expansion that I. Prigogine in Belgium and L. Leopold developed in the USA. In this article a specific topic is explored briefly: The ergodic nature of the energetic processes in the natural flows, its relation with the entropy in the "dynamic equilibrium" and its application to the elucidation of the problem of the transversal distribution of speeds in the rivers, of great importance for the study of the environmental impacts in these bodies of water. The main result of this analysis is to verify the "universal" nature of the model proposed by L. Prandtl at the beginning of the 20 th century.
\end{abstract}

Volume 2 Issue 2 - 2018

\author{
Constaín AJ \\ FLUVIA/Hydrocloro Technologies, Bogotá, Colombia
}

Correspondence: Constaín AJ, FLUVIA/Hydrocloro

Technologies, Bogotá, Colombia,

Email alfredoconstain@fluvia.co

Received: April 22, 2018 | Published: April 30, 2018

\section{The work of I. Prigogine about irreversible processes in fluids}

I. Prigogine (Moscow 1917, Brussels 2003, Nobel Prize for Chemistry in 1977) and his school at the Free University of Brussels extended the scope of thermodynamics beyond equilibrium and systematized the analysis of irreversible, real situations. Regarding the nature of the turbulence, its main result is related to the principle of Minimum entropy production in systems close to equilibrium (as in the case of natural channels in "stable state") and its consequence of "maximum entropy", concordant with external restrictions. According to the statistical definition of L. Boltzmann (Vienna 1844, Duino 1906) for this particular condition, in all the phase space of the system there is an equal probability for the events that are identified there, including naturally those of potential energy. A fluid in turbulence, evolving in "stable state" then presents the remarkable condition of "Equiprobability" for the energetic events in the considered volume. ${ }^{1,2}$

\section{Entropy, probability and ergodicity}

If in the previous conditions the phase space of the System has all its points with equal probability, then from the point of view of energy there is "ergodicity", that is to say that the system performs the exploration of all the points of the phase space in a finite time; from the perspective of Statistical Mechanics this can be done only with a "Markovian" scheme as simple as possible, based on only two decision alternatives (left or right) in a one-dimensional dynamic, that is, a Brownian mechanism. Prigogine then demonstrates that the diffusive movement consistent with the Fick- Brown mechanism is the only way in which the kinetic and potential movements in a fluid develop, attending to the most general thermodynamic conditions, independently of any other restriction. This means that the Brownian movement is the natural way in which the irreversibility of the turbulence develops, in all cases. ${ }^{3,4}$

\section{The normal distribution of probability}

From the perspective of the theory of random functions, a mechanism that is totally erratic, that is to say that all the contributing factors of the movement are independent and small, then the "central limit theorem" is valid, in which the different distributions of Probability that govern such movements (whether normal or not) converge in a final normal distribution. Therefore the turbulence movements, totally heterogeneous (even in the macroscopic fillets, since any potential movement derives in Brownian trajectories), can be described by a normal distribution, being also congruent with the Fickian nature of the same. Nature then chooses the simplest and most uniform statistical description (normal law) for the most complex phenomenon (turbulence). ${ }^{5,6}$

\section{Prandtl's work for natural streams}

\section{The foundation of Prandtl's curve}

L. Prandtl (Freising 1875, Gottingen 1953), a brilliant German engineer and scientist, developed the approach to the movement of fluids in the presence of viscosity. Based on its concept of the logarithmic relationship for this purpose, He proposed the following curve, which describes the distribution of the longitudinal velocities distributed along the transversal axis (width) Figure 1. This curve, initially developed for ideal rugose tubes, was then extrapolated to turbulent channels. UL is the longitudinal speed measured in the partial width (transverse) WL. Umxm is the maximum speed measured at the center of the flow, that is, a partial (half) width of stream, W/2. The approximate equation that describes it has the following form:

$$
\frac{U L}{U m x m}=a * L n\left(b * \frac{W L}{\frac{W}{2}}+c\right)+d * \frac{W L}{\frac{W}{2}}+e
$$


The formula approximate se pueden hallar excel que los valores numéricos que ajustan las constantes los datos experimentales son, ${ }^{7}$

$$
\frac{U L}{U m x m}=0.25 * L n\left(1.73 * \frac{W L}{\frac{W}{2}}+0.0123\right)+(-0.245) * \frac{W L}{\frac{W}{2}}+1.1
$$

The first addition of the right member incorporates the effect of the viscosity that predominates in the border (wall) of the bed and fades towards the center. The second summation includes the linear effect of the free-flowing viscosity near the center of the channel. The parameters $a, b, c, d$ and e are experimental adjustments of the equation. This relationship has a very great importance, since based on it, it is possible to base the calculations of the transverse diffusion of solutes (contaminants) in the flows subject to environmental threat.

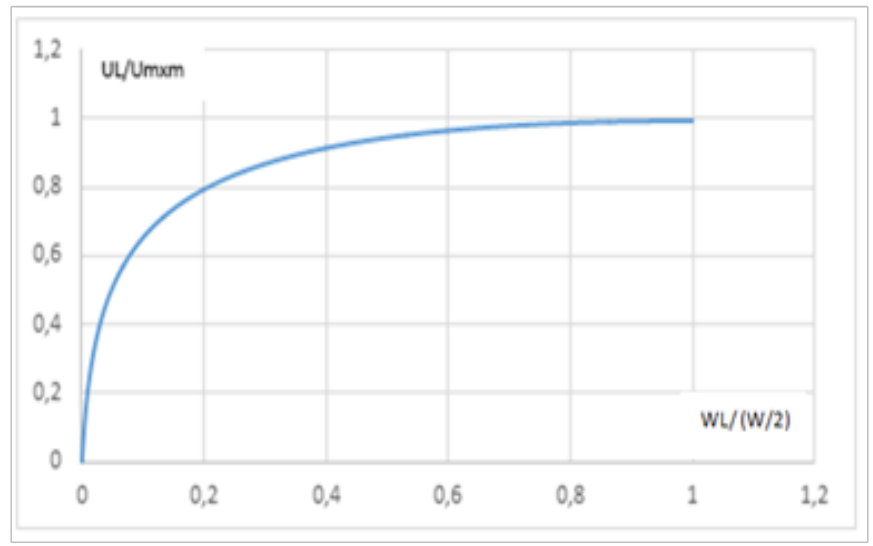

Figure I Prandtl's velocity traverse distribution.

\section{The nature of Prandtl's curve}

Actually, the Prandtl curve is a simplified pictorial representation of a "random function", that is, the infinite set of random magnitudes that are associated with the possible realizations (results) of that function, each with its own probability distribution (superimposed curves in brown) that develop around the average curve (blue). ${ }^{8}$ Figure 2. Then, this curve thus drawn represents both the different, nonunivocal realizations that describe the innumerable different links that condition the phenomenon (brown curves); as the total representation of the "statistical regularity" (blue curve) derived from the so-called "law of large numbers", for frequent random events. ${ }^{9}$ This is the actual effect of the turbulence in the flow, and the actual realization of the longitudinal velocity vectors along the width of said flow.

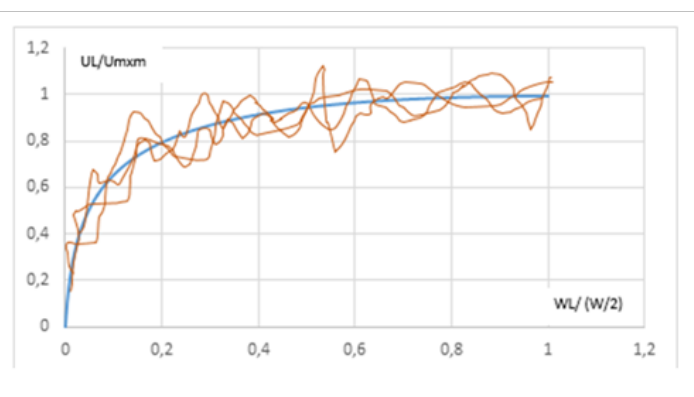

Figure 2 Possible results arranged around the mean.

\section{Prandtl's curve is a general description of velocity in turbulent flows}

\section{The longitudinal velocities lateral distribution in streams}

This concept is described in Figure 3.

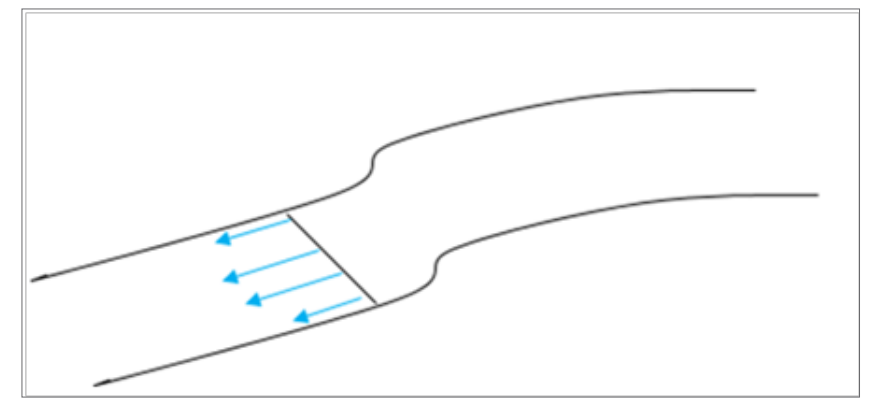

Figure 3 Velocity distributions in a stream.

\section{Several models to represent this distribution}

Despite its quite frequent use in river engineering tasks, the validity of the Prandtl curve and equation (1) is not recognized as a general phenomenon, in fact many different models have been proposed that aim to describe this distribution with "better accuracy" than (1). ${ }^{10}$ This would be plausible if the Prandtl curve were not may be recognized as the only possible one within the physical foundation of the phenomenon. This recognition is presented in the following way.

\section{Leopold's view of streams in "Steady state": Principle of constancy of velocity in flows}

L. Leopold (Albuquerque 1915, Berkeley 2006) was likewise an eminent American scientist who contributed to successfully apply the ideas of I. Prigogine to river hydraulics. It is due to the observation that, "in a natural flow in" stable state ", the average velocity is the same at birth and the delta", that is, the "constancy of the average velocity in natural flows (principle of Leopold).

\section{Thermodynamic explanation of Leopold's Principle}

Though Leopold did not base his Principle more than on the experimental observations on a large number of rivers in the USA, it is not difficult to show that this statement is derived from the principles revealed by I. Prigogine (and used also by Leopold). ${ }^{11}$ Thus, based on the principle of "Equiprobability" for flows in "steady state" close to equilibrium, it can be seen that if the potential energy is distributed throughout the volume of the system in an "equiprobable" way, then the transfer of mass from one point to another of this phase space is equivalent for all points, and therefore the velocities are equivalent. The constancy of the average velocity in "the whole flow" (longitudinally) is then given as a consequence of the thermodynamics of the irreversible processes.

\section{Application of Leopold's principle to fluvial dynamics in transverse sense}

If in a large distance the principle of Leopold is valid, much more so in a short distance. Thus, not only in the longitudinal sense is the validity of the flow rate in "Equiprobability" valid but also in transverse direction. Therefore, across the width distribution of longitudinal speeds is uniform, i.e. it is a straight line (constancy of velocities in this axis), as in upper Figure 4. If you take into account the viscosity 
acting in the vicinity of the wall, then this distribution deflects as in lower Figure 4. Then, the Prandtl curve is actually a consequence of the Constancy Principle of velocity in the thermodynamic system considered, and in this sense it is a general definition, which is not met for other models of different velocity distribution.

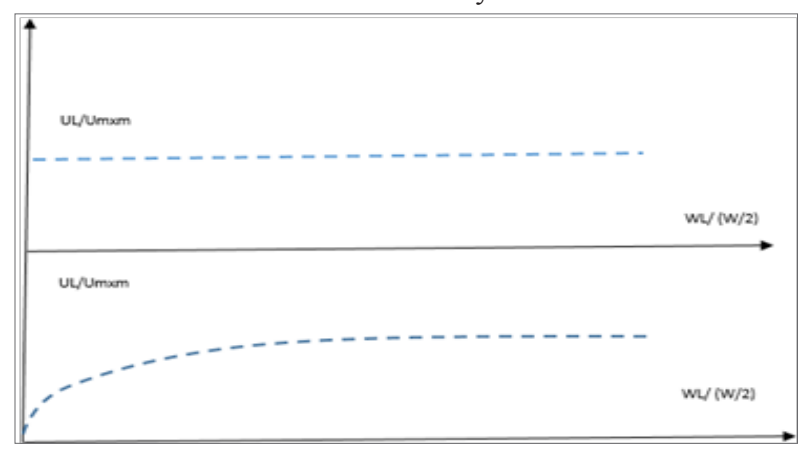

Figure 4 Leopold principle and prandtl deflection

\section{Normal (Gaussian) nature of Prandtl curve}

The next step is to verify that the structure of the data associated with the curve corresponds to a normal distribution. To do this verification, we start from the known fact that in a distribution of this type the value of the "Mathematical Hope" (general average) coincides with the value of the "median" (that is, the value of the abscissa that separates equal of probability, on each side of the average). To calculate the Mathematical Hope of this random quantity, the following equation is used for random quantities:

$$
M\{x\}=\frac{\int_{-\infty}^{\infty} x * \psi(x) d x}{\int_{-\infty}^{\infty} \psi(x) d x}
$$

Applying this definition to the concrete case of equation (1) we have:

$$
M\left\{W L /\left(\frac{W}{2}\right)\right\}=\frac{\int_{-\infty}^{\infty} W L /\left(\frac{W}{2}\right)^{*\left(\frac{U L}{U m x m}\right) d} W L /\left(\frac{W}{2}\right)}{\int_{-\infty}^{\infty}\left(\frac{U L}{U m x m}\right) d W /\left(\frac{W}{2}\right)}
$$

This calculation can be done in an approximate way using an Excel routine on the concrete curve, equation (2):

This calculation can be done in an approximate way using an Excel routine on the concrete curve, equation(2)

$$
M\left\{W L /\left(\frac{W}{2}\right)\right\} \approx 0.544
$$

The function UL/Umxm is also calculated for the mean value of the abscissa, WL / $(\mathrm{W} / 2)=0.544$ :

$$
\frac{U L}{U m x m}=0.25 * \operatorname{Ln}(1.73 * 0.544+0.0123)+(-0.245) * 0.544+1.1 \approx 0.954795
$$

Now, in relation to the curve of Figure 1, the notable values are drawn and the areas on the side and side of the mean value of the independent variable (abscissa) are calculated Figure 5.

Using the Excel tool to calculate the area under the curve, for the curve in question (with all the decimal expansion) we have:

$$
A 1 \approx 0.427441154 \text { And } A 2 \approx 0.447714436
$$

Now, if you calculate the ratio of these two areas, you have:

$$
\frac{A 1}{A 2}=\frac{0.427441154}{0.447714436}=0.954718275
$$

If this value is observed, it coincides with the value of the function of the mean value. This coincidence can be explained by examining Figure 6, in which a normalized Gaussian curve is drawn at maximum value $=1$. In this unbiased curve we have that the function is equal to 1 , also that the ratio of the areas on each side of the mean is equal to 1. If we take into account that the values of the Prandtl distribution, equation (2) and Figure 3, is of Brownian (Gaussian) nature but with a biased characteristic (due to presence of viscosity), then the ratio of the areas also equals the function and reflects the observed bias.

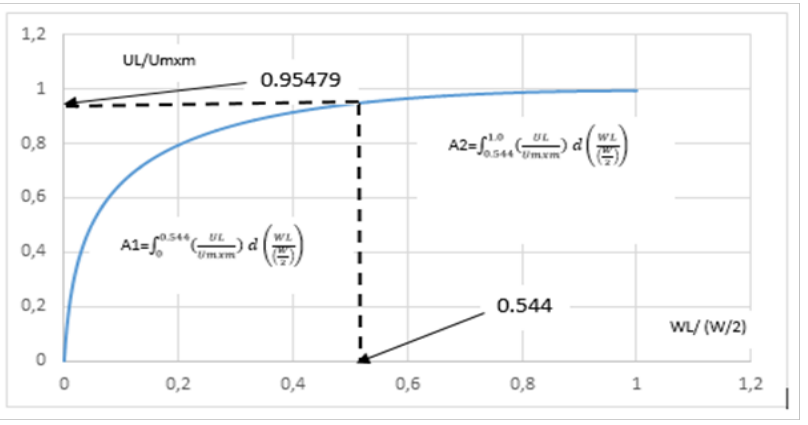

Figure 5 Probabilities about the mean value and important values.

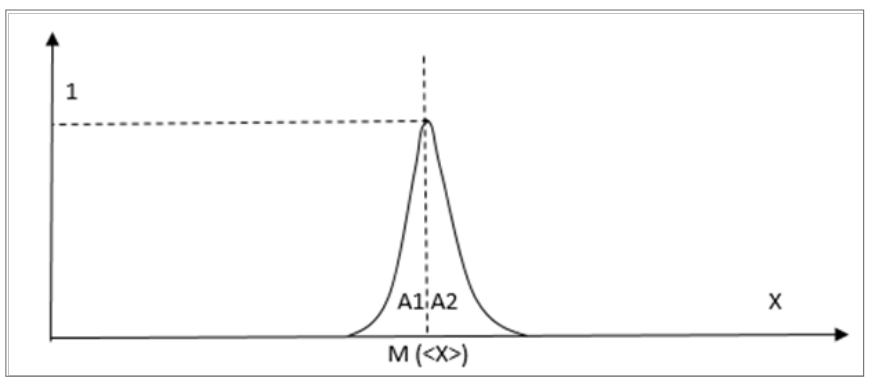

Figure 6 Normalized Gaussian curve characteristics

\section{Conclusion}

The lateral distribution of the longitudinal velocities in the natural channels, initially described by L. Prandtl, is not only the manifestation of the effect of the viscosity by the presence of the walls of the bed on the flow movement, but the imposition of conditions general by the thermodynamics of irreversible processes. From this perspective, it is not a distribution, but the only possible one, according to these general restrictions. It is shown by the extensive works of I. Prigogine that the Normal distribution (Gauss) is a basic feature of the evolution of irreversible natural phenomena, manifesting itself in ergodic processes. As a consequence of the thermodynamic conditions on the entropy in the flows in "dynamic equilibrium" (stable state), the average speed in the natural channels must tend to be a constant value, attending to the "Equiprobability" of events. It is shown in this article how the specific conditions of the normal distribution are fulfilled for the Prandtl curve (equation), especially the convergence of the mean 
value and the median.

\section{Acknowledgements}

None.

\section{Conflict of interest}

The author declares there is no conflict of interest.

\section{Referenecs}

1. Prigogine I, Stengers I. The New Alliance: metamorphosis of science. Madrid, Spain: Alianza; 1997:360.

2. Leopold L, Langbein W. The concept of entropy in landscape evolution. Washington: GS professional paper 500-A. 1962:1-26.

3. Prigogine I, Nicolis G. The structure of the complex. Madrid, Spain Alianza; 1994:392.

4. Bendat J, Piersol A. Random Data: Analysis and measurement procedures. New York: Wiley-Interscience; 1986:640.

5. Mandel J. The statistical analysis of experimental data. New York: Dover;
1962:432.

6. Spiridonov V, Lopatkin A. Mathematical treatment of physicochemical data. Mir Moscow, Russia: 1972:207.

7. Constaín A. Una verificación approximate de la Ley de distribución lateral de velocidades en flujos turbulentos de cauces naturales (Prandlt) en Equilibrio dinamico mediante trazadores. Revista ACODAL No. 238; 2015 .

8. Pugachev V. Introduction to the theory of probabilities. Mir, Moscow; 1973:492.

9. Zajarov V, Sebastianov B, Chistiakhov V. Probability Theory. Mir Moscú. 1977.

10. Seo I, Baek K. Estimation of the longitudinal dispersion coefficient for streams. Belgium: River Flow; 2002:130.

11. Leopold L, Maddock T. The hydraulic geometry of stream channels and some physiographic implications. Washington: GS professional paper $252 ; 1953: 57$. 